\title{
Individual Behavioral Responses to Hypothalamic Stimulation Persist Despite Destruction of Tissue Surrounding Electrode Tip ${ }^{1}$
}

\author{
SUSAN E. BACHUS AND ELLIOT S. VALENSTEIN \\ Department of Psychology, University of Michigan, Ann Arbor, MI 48109
}

Received 23 April 1979

\begin{abstract}
BACHUS, S. E. AND E. S. VALENSTEIN. Individual behavioral responses to hypothalamic stimulation persist despite destruction of tissue surrounding electrode tip. PHYSIOL. BEHAV. 23(3) 421-426, 1979.-Behavior evoked by hypothalamic stimulation differs among animals, although their electrode placements appear histologically to be identical. These differences have been attributed to selective activation of functionally discrete neural circuits that are in close proximity. We report here, however, that in rats initially exhibiting "stimulus-bound" drinking, this behavior persists following either a series of progressively larger lesions, or a single large lesion, around the electrode tips. Similar results were obtained in rats afforded minimal stimulation experience prior to the lesions. These results suggest that individual differences in response to hypothalamic stimulation cannot be attributed solely to variations in the neuroanatomical locus of stimulation.
\end{abstract}

Stimulus bound drinking $\quad$ Lateral hypothalamus Electrical stimulation Electrolytic lesions Individual differences Experience Neuroanatomical locus

STRIKING individual differences in the behavior evoked by electrical stimulation of the lateral hypothalamus (ESLH) have been observed $[1,4,7,8,9]$. In response to ESLH, individual animals may, for example, either eat, drink, groom, carry objects, attack other animals, or exhibit heightened sexual activity, while other animals display only locomotor activation [8]. Although some of these differences in stimulus-bound behavior may be attributable to gross differences in electrode placements, or to factors associated with the testing conditions, it has been demonstrated that even with identical testing procedures and electrode placements that are indistinguishable, animals vary in their response to ESLH [2]. It has been argued that elicitation of different behaviors by identically placed electrodes may be attributed to the differential activation of anatomically overlapping, but functionally distinct, neural circuits $[3,6,9]$ or to individual variability in localization of the critical substrates [6]. The observation that many animals with multiple electrodes display the same behavior in response to stimulation at different hypothalamic sites, even when this behavior is an unusual one, suggests, however, that individual differences cannot be attributed solely to subtle variations in electrode placement [8].
Additional evidence of the importance of individual differences in determining the behavior evoked by stimulation was derived from data obtained with "moveable electrodes" which were advanced in a dorsal to ventral plane through the brain in rats [9]. Animals displaying eating and drinking continued to display this behavior even after the electrodes were advanced as much as $1.5 \mathrm{~mm}$ through the hypothalamus, while in others, no eating or drinking was evoked at any of the sites. While these results suggest an important role for individual response tendencies, at least two alternative interpretations remain. First, due to the inability to specify the extent of current spread, the possibility exists that the same "critical structures" were activated after the electrodes were moved. Second, animals were tested extensively and at relatively high current levels after each small $(0.133 \mathrm{~mm})$ incremental movement. This procedure may have gradually broadened the substrate capable of evoking a particular response, for example, through stimulation of overlapping fields. This latter possibility is supported by the subsequent finding in monkeys that drinking was evoked at an increasing proportion of sites following stimulation at sites that evoked drinking [1]. In order to minimize the possibility that the stimulation initially evoking a behav-

${ }^{1}$ This work was supported by NIMH Grant MH20811-03. We thank B. Martonyi for technical assistance, S. Winans for histological advice, and R. Katz, C. Boast, and T. Robinson for their helpful comments. 
ior was activating the same "critical structures," and to determine whether the extent of tissue capable of evoking a behavior could be influenced by prior stimulation experience, we employed a different strategy. Animals which exhibited stimulus-bound drinking were retested following the production of large electrolytic lesions around the tips of the electrodes, both under conditions of considerable stimulation experience prior to the lesions (Experiment 1), and with minimal pre-lesion experience (Experiment 2).

\section{GENERAL METHOD}

Bipolar electrodes were implanted unilaterally in the lateral hypothalamus of mature male and female rats $(250-610 \mathrm{~g}$ at time of surgery) of Sprague-Dawley (Holtzman Co., Madison, WI) and hooded, Long Evans (Simonsen Co., Gilroy, CA) strains, under Equithesin anaesthesia. Electrodes were twisted bipolar stainless steel wires (No MS 303/1, $0.25 \mathrm{~mm}$ dia., Plastic Products Co., Roanoke, VA), bared of insulation only at the adjacent tips. Stereotaxic coordinates were $3.5 \mathrm{~mm}$ posterior to bregma, $1.5 \mathrm{~mm}$ to the right of the midsagittal suture, and $8.25 \mathrm{~mm}$ below the surface of the skull with the skull held level between bregma and lambda.

\section{EXPERIMENT 1}

Following recovery, rats were placed in a $20.5 \times 26.5 \times 42.5$ $\mathrm{cm}$ Plexiglas chamber with access to a drinking tube, and stimulated with $20 \mathrm{sec}$ trains of $60 \mathrm{~Hz}$ sine waves from a constant current source, alternating with $20 \mathrm{sec}$ inter-trial intervals. Rats $(\mathrm{N}=27)$ that consistently drank water during ESLH were selected for Experiment 1 from a much larger group that had comparably placed electrodes, but did not drink when stimulated. Experimental animals were placed on a schedule of "threshold testing" which enabled us to determine the minimum stimulation current that evoked drinking. The threshold test consisted of a "staircase titration" method in which the intensity was raised in $1 \mu \mathrm{A}$ steps if no drinking was observed, but was repeated at the same intensity when drinking occurred. If drinking did not recur, the intensity was raised again. When drinking recurred at the same intensity, the current was decreased $3 \mu \mathrm{A}$, and the process repeated twice more. The three intensities at which drinking occurred twice in succession were averaged for the "daily threshold." During the first few tests, the thresholds tended to decline prior to stabilizing at some lower asymptote level, which varied between $1.0-28.0 \mu \mathrm{A}$ in individual animals.

Following threshold stabilization (defined as 3 consecutive daily tests with a threshold range not exceeding 2 $\mu \mathrm{A})$, a series of progressively larger electrolytic lesions were made around the electrode tips by passing direct current for $15 \mathrm{sec}$ between one electrode wire (either pole A or B) and an indifferent, cathodal contact attached to the ear. Lesions are designated as follows: Lesions 1 and $2(0.25 \mathrm{~mA}, \mathrm{~A} \& \mathrm{~B}$, respectively); Lesions 3 and $4(0.50 \mathrm{~mA}, \mathrm{~A}$ and $\mathrm{B}$, respectively); Lesions 5 and $6(1.0 \mathrm{~mA}, \mathrm{~A}$ and $\mathrm{B}$ respectively); Lesion 7 ( $2.0 \mathrm{~mA}$, pole $A)$. Lesions were made while the rats were lightly anesthetized with ether.

Testing was resumed three days after each lesion. Typically, higher currents were required after a lesion. Current was raised in $2 \mu \mathrm{A}$ increments for thresholds in the $50-200 \mu \mathrm{A}$ range, and in $5 \mu \mathrm{A}$ increments when current levels over $200 \mu \mathrm{A}$ were required.
Rats were divided into 3 groups in order to assess the significance of additional stimulation experience between lesions and the time interval between successive lesions. Animals in Group $1(\mathrm{~N}=12)$ were given a series of threshold tests after each lesion. Generally, the next lesion was not made until after the threshold was judged stable. In Group 2 $(\mathrm{N}=9)$ there were no additional threshold tests administered until after the completion of Lesion 4. Time intervals between Lesions 1, 2, 3, and 4 were equal to the average intervals in Group 1, ranging from 9 to 22 days. Following the completion of threshold testing after Lesion 4 , Lesions 5 and 6 were made and behavioral tests were administered after each of these lesions. In Group $3(\mathrm{~N}=6)$ Lesions 3 and 4 were both produced immediately after the completion of the initial series of threshold tests. Lesions 5 and 6 were made after threshold data had been obtained. In 2 rats of this group Lesion 7 was also produced.

\section{Results}

In all rats tested, drinking was still elicited by ESLH following Lesion 4 , regardless of whether the lesions were made serially or in one stage, or whether or not intervening stimulation experience was given. (Electrodes were dislodged before the fourth lesion was produced in 4 animals in Group 1.) In most cases, the current threshold was elevated following each lesion, but it was still possible to obtain stable threshold data. In those animals given Lesion 6, drinking also could still be evoked, but in two instances, not with sufficient consistency to determine thresholds with our protocol. Thresholds rose from an average of $6.9 \mu \mathrm{A}$ before lesions to $23.7 \mu \mathrm{A}$ after Lesion $1(\mathrm{~N}=12), 84.3 \mu \mathrm{A}$ after Lesion $4(\mathrm{~N}=23)$, and $165.1 \mu \mathrm{A}$ after Lesion $6(\mathrm{~N}=11)$. Of the three rats given Lesion 7, two drank at an average of 87.5 $\mu \mathrm{A}$, while the other no longer drank. One animal tested within 30 min following Lesions 3 and 4 demonstrated a comparable threshold elevation, from 18.0 to $80.0 \mu \mathrm{A}$. Figure 1 presents representative results from Groups 1 and 2.

\section{EXPERIMENT 2}

In order to determine whether the series of stimulation tests given to all animals prior to the production of lesions could have influenced the results, 12 naive rats were tested, using the methods described in Experiment 1, except that these animals received minimal stimulation experience prior to the lesions. One week after electrode implantation, animals received $20 \mathrm{sec}$ stimulation trains at intensities within the range of pre-lesion thresholds of animals in Experiment 1. In several rats drinking was observed during the first stimulation train. All animals received only the minimal number of stimulation trains judged necessary to confirm that drinking was evoked by stimulation. Thus, prior to the production of lesions most animals $(N=9)$ received no more than 4 stimulation trains and 3 received only 2 trains. The currents used varied between 2-18 $\mu \mathrm{A}$. In 8 rats, Lesions 3 and 4 were then produced either in one stage (Lesions $3+4$ ), or serially (Lesions $1,2,3$ and 4), prior to the initiation of threshold testing; in 4 rats, Lesions 5 and 6 were produced in a single stage prior to testing. Threshold testing was performed over a two week period following the lesions.

\section{Results}

In spite of the minimal stimulation experience, all rats 


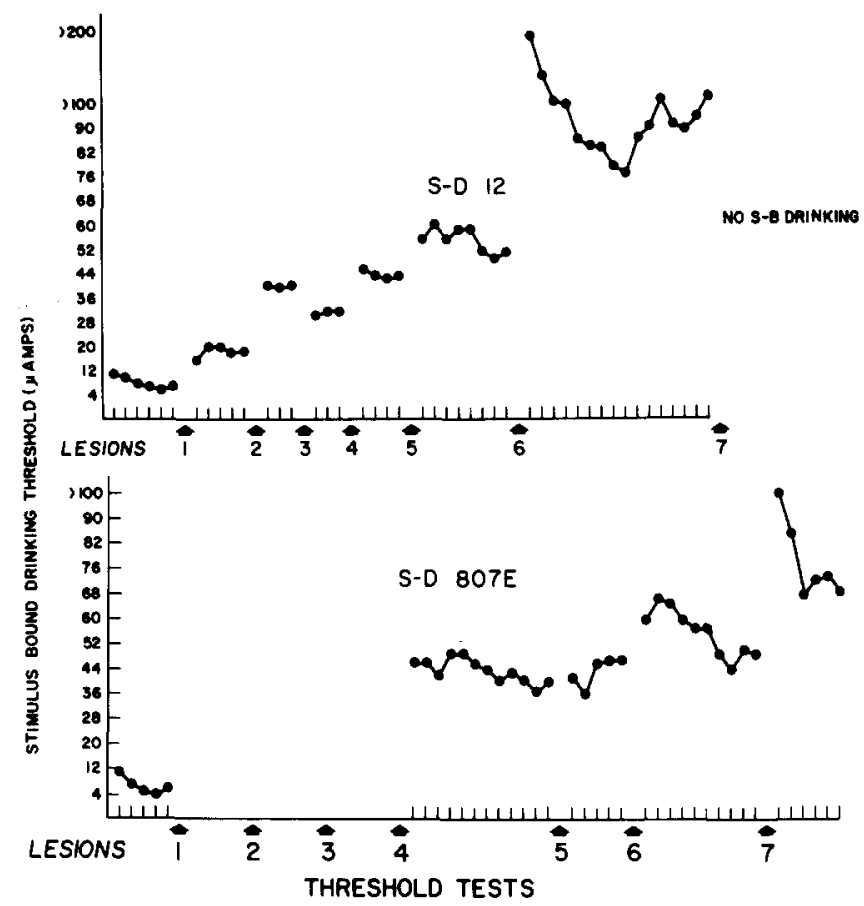

FIG. 1. Daily thresholds for elicitation of drinking by electrical stimulation of the lateral hypothalamus; preceding, and subsequent to a series of progressively larger lesions produced around the tip of the stimulating electrode. (Lesions 1 and 2 were produced with 0.25 $\mathrm{mA}$ direct current for $15 \mathrm{sec}$ through poles $\mathrm{A}$ and $\mathrm{B}$, respectively of the bipolar electrode; Lesions 3 and 4 with $0.50 \mathrm{~mA}$ for $15 \mathrm{sec}$ through poles $A$ and $B$ respectively; Lesions 5 and 6 with $1.0 \mathrm{~mA}$ for $15 \mathrm{sec}$ through poles $A$ and $B$ respectively; and Lesion 7 with $2.0 \mathrm{~mA}$ for $15 \mathrm{sec}$ through pole A.) Animal S-D 12 was tested following each lesion. Drinking was consistently evoked, generally at progressively higher thresholds, following each of Lesions 1-6, but was abolished by Lesion 7. Animal S-D 807E received the same series of lesions, separated by time intervals comparable to those for S-D 12, but was not retested until after Lesion 4 . When retested, drinking was still obtained, with a threshold elevation comparable to that seen in S-D 12. Drinking was still reliably evoked in S-D 807E following Lesion 7.

continued to drink in response to stimulation after the lesions. For the 8 animals receiving Lesions 3 and 4, current required rose from a screening estimate of $6.8 \mu \mathrm{A}$ prior to the lesions to an average threshold of $48.3 \mu \mathrm{A}$ after serial Lesions $1-4$ and $118.5 \mu \mathrm{A}$ after single-stage Lesions $3+4$. Figure 2 depicts the results of the 4 rats that received the larger single-stage lesions (Lesions $5+6$ ). The currents required to evoke drinking after the lesions were very high and the thresholds were not stable, but there was no doubt that stimulation still produced the behavior in all 4 cases.

Histological examination suggested that the single-stage lesions produced greater tissue damage than did the serial lesions. Since we observed coagulated tissue around the electrode tips we inferred that tissue damaged by prior lesions may have impeded current spread, during subsequent production of lesions, as well as during threshold testing. The greater damage caused by single-stage relative to serial lesions may have been responsible for the greater threshold elevations following single-stage lesions.
Damage was completely reconstructed for the 4 rats receiving Lesions $5+6$ in Experiment 2 . In all 4, damage spread over $3 \mathrm{~mm}$ along an anterior-posterior axis through the medial forebrain bundle. In 3 of these 4 animals, the maximum damage was cylindrical, extending over $1.0 \mathrm{~mm}$ in length, with a diameter greater than $1.0 \mathrm{~mm}$. The fourth, slightly smaller lesion, was found in the rat with the lowest post-lesion threshold. Figure 3 illustrates the extent of the anatomical damage on a reconstruction of frontal sections through the electrode tract in one animal. Although the threshold was raised to $130 \mu \mathrm{A}$ after Lesions $5+6$, from an initial estimate of $5.0 \mu \mathrm{A}$ (based on 2 stimulation trains), drinking was still reliably evoked by ESLH.

\section{DISCUSSION}

The results of both Experiments 1 and 2 indicate that after extensive damage to the hypothalamic circuitry surrounding the tip of the electrode, it was still possible to evoke 


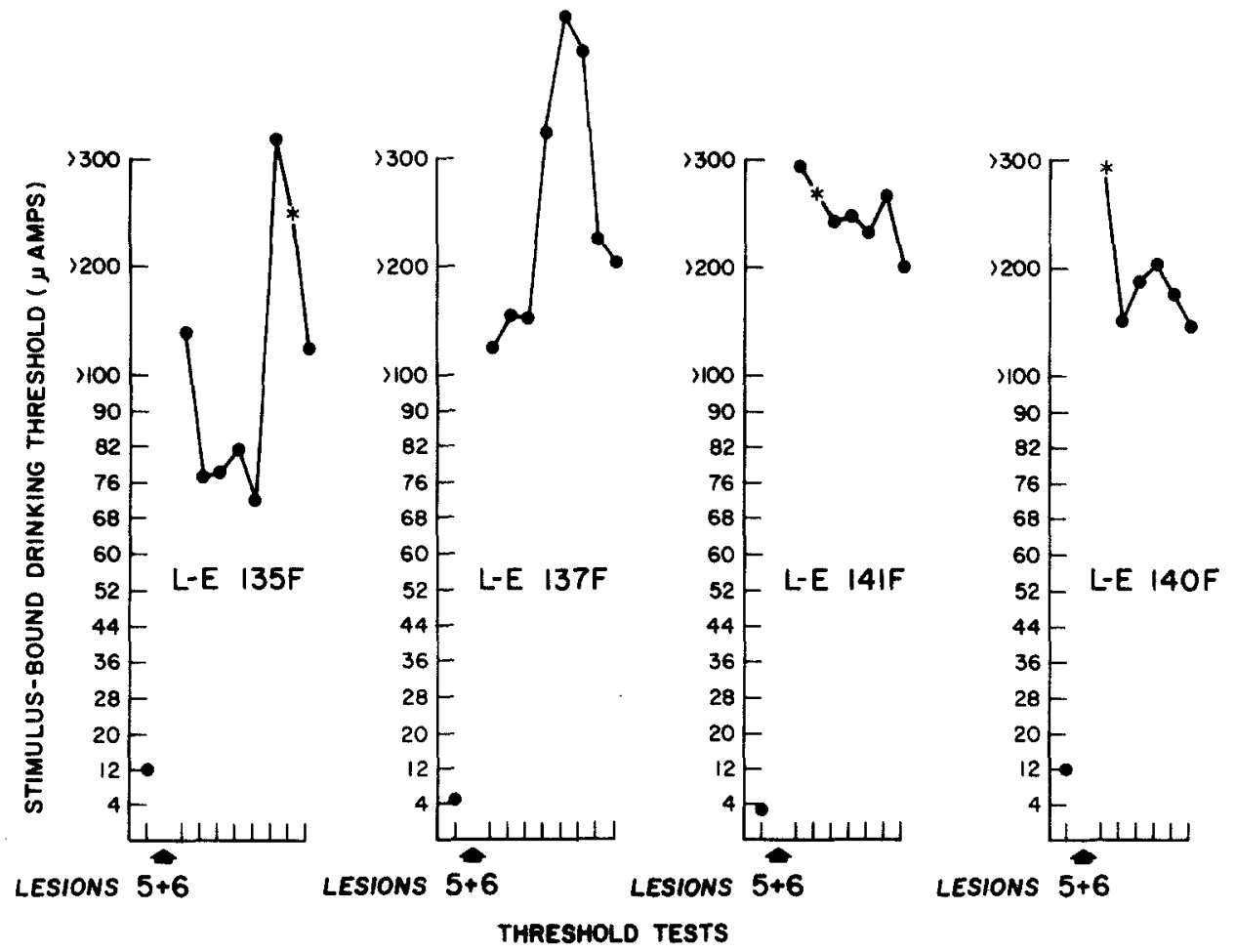

FIG. 2. Daily thresholds for elicitation of drinking by lateral hypothalamic stimulation in rats given minimal stimulation experience prior to producing large lesions around the electrode tip. The lesions were made in one stage by passing $1.0 \mathrm{~mA}$ of direct current through each of the two poles of the stimulating electrode. The pre-lesion "threshold" is based on $2-4,20 \mathrm{sec}$ trains, the only stimulation experience the animals received prior to the lesions. The asterisk(*) represents average intensities evoking drinking when there was not sufficient consistency to meet our criterion for a threshold.

stimulus-bound drinking in animals which initially displayed this behavior. The persistence of stimulus-bound drinking in the experimental animals following the production of large lesions supports the conclusion that individual differences in response to ESLH cannot be explained by subtle differences in which of many anatomically intertwined circuits was activated. To appreciate the significance of these results, it is necessary to consider the low stimulating current levels that were initially sufficient to evoke drinking in many animals, the use of bipolar electrodes with adjacent tips, and the large area of destruction around the electrode tips produced by the larger lesions. Under these circumstances, it is likely that the neuronal elements that were directly activated by the initial stimulation were completely destroyed, or at the very least, the circuitry was radically altered. Furthermore, the results in Experiment 2, where minimal pre-lesion stimulation experience was provided, indicate that the elicitation of ESLH-evoked drinking over a considerable extent of tissue does not depend upon changes induced by a long regimen of stimulation.

The importance of individual differences in determining the response to ESLH is supported by some additional observations. First, it should be recalled that the subjects in Experiments 1 and 2 were selected from a much larger pool of animals. The animals that were not used as subjects had comparably placed electrodes, but they only displayed locomotor-exploratory behavior even though stimulation current levels were gradually raised up to intensities that disrupted behavior. Thus in animals that initially do not display stimulus-bound behavior, extending the neural field activated by the stimulation is not effective, whereas in animals that initially do exhibit such behavior, destruction of the stimulated tissue does not eliminate the behavior. That individual characteristics influence response to ESLH is also evidenced by our observation of a strain difference in probability of hypothalamic stimulation eliciting eating and drinking. Of the two strains screened for subjects in these experiments, only $10-30 \%$ of the Sprague-Dawley rats, as contrasted with $80-90 \%$ of the Long Evans rats, displayed eating or drinking in response to ESLH. The results of Experiments 1 and 2 along with the arguments presented above lead us to conclude that some animals are predisposed to drink in response to stimulation within a relatively large hypothalamic area.

The results reported here are concerned only with stimulus-bound drinking, but in pilot studies comparable results were obtained with animals that ate, or licked at the walls of the testing arena, in response to ESLH. Other behaviors, however, have not been studied. The determination of the factors which influence the predisposition of animals to respond to ESLH with a particular behavior is undergoing further investigation in this laboratory. 


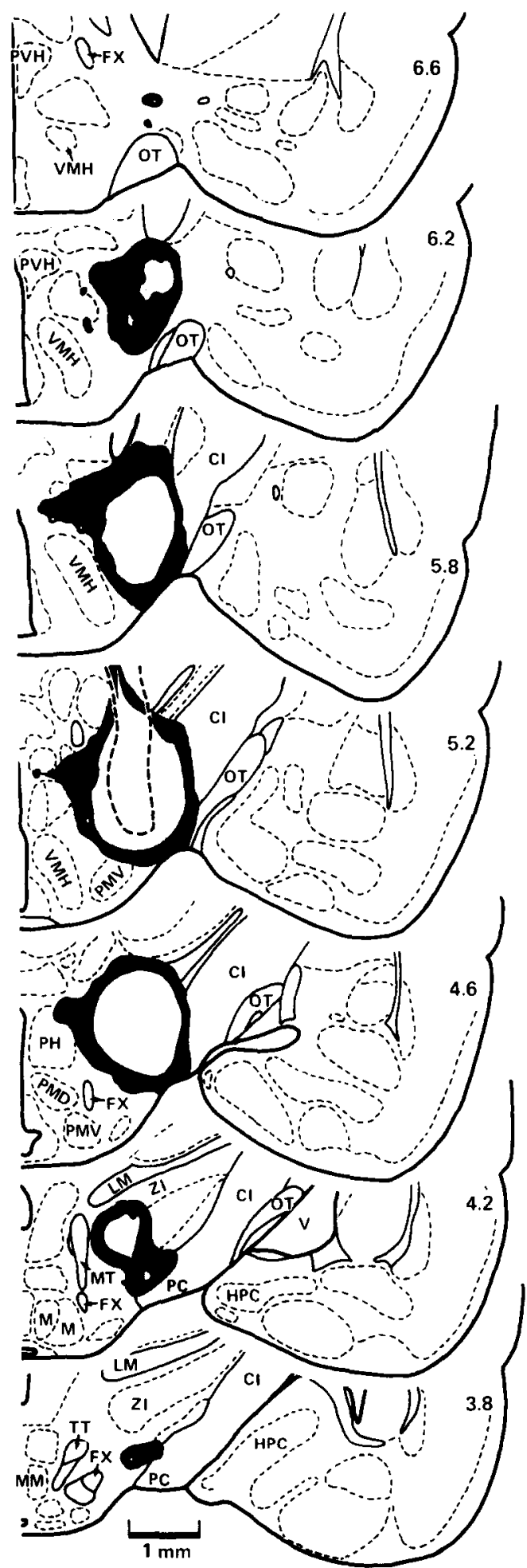

FIG. 3. Histological reconstruction of the damage produced by Lesion $5+6$ in Animal L-E 137F, whose post-lesion thresholds for elicitation of drinking are summarized in Fig. 2. Although maximum tissue damage around the electrode tip extended over $1 \mathrm{~mm}$ in diameter, and over $1 \mathrm{~mm}$ in an anterior-posterior (A-P) plane, drinking was still evoked reliably in this rat up to the time of sacrifice and perfusion. Some cell damage was detectable over a $3.0 \mathrm{~mm}$ A-P extent. The dashed line indicates a representative electrode tract, from an unlesioned rat with comparable testing experience. Solid shading around the border of the lesion represents an estimation of the extent of gliosis. Damage was plotted from $80 \mu$ sections, stained with Cresyl-violet. Abbreviations: Cl-internal capsule; FXfornix; HPC-hippocampus; LM-medial lemniscus; MM-medial mamillary nucleus; MTmamillothalamic tract; OT-optic tract; PC-cerebral peduncle; $\mathrm{PH}$-posterior nucleus of hypothalamus; PMD-dorsal premamillary nucleus; PMV-ventral premamillary nucleus; V-ventricle; ZI-zona incerta. [5] 


\section{REFERENCES}

1. Bowden, D. M., T. Galkin and H. E. Rosvold. Primate drinking system as defined by electrical stimulation of the brain (ESB). Physiol. Behav. 15: 103-111, 1975.

2. Cox, V. C. and E. S. Valenstein. Distribution of hypothalamic sites yielding stimulus bound behavior. Brain Behav. Evol. 2: 359-376, 1969.

3. Milgram, N. W., M. Devor and A. C. Server. Spontaneous changes in behavior induced by electrical stimulation of the lateral hypothalamus in rats. J. comp. physiol. Psychol. 75: 491$499,1971$.

4. Panksepp, J. Aggression elicited by electrical stimulation of the hypothalamus in albino rats. Physiol. Behav. 6: 321-329, 1971.
5. Pellegrino, L. J. and A. J. Cushman. A Stereotaxic Atlas of the Rat Brain. New York: Meredith, 1967.

6. Roberts, W. W. Are hypothalamic motivational mechanisms functionally and anatomically specific? Brain Behav. Evol. 2: $317-342,1969$.

7. Valenstein, E. S. Behavior elicited by hypothalamic stimulation: A prepotency hypothesis. Brain Behav. Evol. 2: 295-316, 1969.

8. Valenstein, E. S., V. C. Cox and J. Kakolewski. A reexamination of the role of the hypothalamus in motivation. Psychol. Rev. 77: $16-31,1970$.

9. Wise, R. A. Individual differences in effects of hypothalamic stimulation: The role of stimulation locus. Physiol. Behav. 6: 569-572, 1971. 